\title{
An Analysis of the Perspectives of 'MGNREGS' Beneficiaries and Implementing Agencies about the Planning, Implementation and Shortcomings of the Scheme in Telangana State, India
}

\author{
D. Kumara Swamy*, C.V. Hanumanthaiah, P. Parthasarathy Rao, K. Suhasini, \\ V.V. Narendranath and R. Vijaya Kumari
}
Department of Agricultural Economics, College of Agriculture, Professor Jayashankar Telangana State Agricultural University, Rajendranagar, Hyderabad - 500030, Telangana, India

*Corresponding author

\section{A B S T R A C T}

The present paper aimed at identifying and analyzing the opinions of beneficiaries of MGNREGS and its implementing agencies on planning, implementation and hurdles in implementation of the scheme in Karimnagar and Medak districts of Telangana state. For the purpose of the study, two mandals where comparatively highest amount of money was

\section{Keywords}

MGNREGS, Highest Expenditure Mandals (HEMs), Lowest Expenditure Mandals (LEMs), Beneficiaries, Difficulties, Constraints and Job card

Article Info

Accepted: 08 July 2018 Available Online: 10 August 2018 spent by the government on the scheme and two mandals where lowest amount of money was spent were selected from each district. Again from each mandal, two villages were selected randomly. From each selected village, 4 farmers and 4 labourers were selected who were MGNREGS beneficiaries. The perspectives of the sample people were recorded, analyzed and found that planning process in MGNREGS was very good, a majority of $34.4 \%$ of respondents in HEMs and $37.5 \%$ in LEMs of Karimnagar expressed it whereas in Medak, $46.9 \%$ in HEMs, $34.4 \%$ in LEMs felt the same. Regarding implementation, a majority in Karimnagar was not happy with the frequency of payments and staff dedication in implementation in HEMs and it was low in LEMs. Whereas in Medak, both in HEMs and LEMs, they were happy with the frequency of payments. Staff dedication in implementation was at middling level. Effect of social auditing was very high in HEMs and it was moderate in LEMs in both the districts. The major difficulties appeared to be finding out the appropriate and sufficient amount of work for all applicants, preparation of action plans, verification and issuing of job cards, elimination of fake and undeserved candidates respectively in both the districts and when it comes to constraints, illiteracy of the beneficiaries, inadequate professional and administrative staff were found to be the major ones in successful implementation of the scheme and suggested to overcome the same for successful implementation of MGNREGS.

\section{Introduction}

Since its inception in 2006, Mahatma Gandhi National Rural Employment Guarantee
Scheme (MGNREGS) as a single largest employment programme in the world had captured maximum amount of money in the budget allocation and involved larger number 
of beneficiaries than any other scheme in India. This programme has effected agriculture directly and indirectly and brought change in labour supply and effected ground water tables according to various reports.

Over a period of time, the ambit of the scheme has been significantly expanded to private lands of small and marginal farmers to create durable assets.

Together, they constitute about $80 \%$ of total farm holdings and own $40 \%$ of the total cultivable land in the country and thus the programme has touched majority of the rural masses.

It is a significant legislation of our times. It provides livelihood, income and a legitimate chance to lead a descent life and encompassed three basic tasks for empowerment of rural poor viz., reduction of poverty, creation of employment and minimizing inequality.

The legal entitlements to the workers mark a transition from a supply to a demand driven employment programme there by making the overall development delivery system highly responsive and accountable.

Except few draw backs such as less focus on assets with a minimum guarantee quality that has to be created with MGNREGS and lack of attention on upgrading the skills of rural youth so as to enable them to earn more income, it has got appreciation from all sections of contemporary society.

With this background, a study has been conducted purposively in Medak from Central Telangana region and Karimnagar form Northern Telangana region of Telangana state to elicit and analyze the opinions of beneficiaries and implementing agencies of MGNREGS on planning, implementation and shortcomings associated with the scheme.

\section{Objectives}

To analyze the opinion of individual beneficiaries and various agencies related to planning and implementation of the MGNREGS.

To identify and analyze the procedural difficulties and constraints in the implementation of the MGNREGS.

\section{Materials and Methods}

The present study was conducted in Karimnagar and Medak districts of Telangana state (formerly part of Andhra Pradesh) during 2013-14. In each district, two mandals were selected purposively where comparatively highest amount of money was spent and two mandals where lowest amount of money was spent on MGNREGS by the government.

From each selected mandal, two villages were selected randomly and from each selected village, eight beneficiaries of the scheme were selected of which $50 \%$ (four) were farmers and $50 \%$ (four) were labourers (Table 1).

Thus, 32 farmers and 32 labourers from each district were selected and finally it made a sample size of 128 respondents.

Data regarding the opinions of individual sample beneficiaries and representatives of various implementing agencies like District Water Management Agency, Office of the District Chief Planning Officer, Commissionerate of Agriculture and Field Assistants of respective villages, Engineering Consultants, Programme Officers of the MGNREGS of respective districts on planning, implementation, difficulties and constraints associated with the scheme were collected as per the objectives of the study in interview method. A pretested questionnaire specially designed for the purpose was used. 
The data collected thus were analyzed with the help of tabular analysis and statistical techniques like Kendall's rank correlation coefficient (w) test, Mann-whitney U test and Mean standard deviation technique etc, interpreted and drawn conclusions.

\section{Kendall's coefficient of concordance test}

The difficulties involved in implementing the scheme at field level were identified and elicited information on difficulties such as finding out the appropriate work, sufficient work, preparing action plans, arranging funds and facing political pressures etc.

Questions were grouped under 10 headings and MGNREGS implementing staff were requested to rank each constraint.

The entire data were analyzed with the help of simple averages and finally ranks were assigned to each difficulty ranging from 1 to 8 . Then, the test was used to find out whether there was significant agreement among the entire scheme implementing staff. If there was significant agreement, then final ranking can be assigned to constraints.

Kendall's coefficient of concordance test (W) was applied by using the formula:

$$
\mathrm{W}=\frac{12 \sum(\mathrm{Ri}-\mathrm{R})^{2}}{\mathrm{~K}^{2}\left(\mathrm{~N}^{3}-\mathrm{N}\right)}
$$

Where,

$\mathrm{R}=$ Mean of ranks assigned to difficulties

$R_{i}=$ Rank given to $i^{\text {th }}$ difficulty

$\mathrm{K}=$ No. of samples taken (i.e., no. of implementing agencies interviewed)

$\mathrm{N}=$ No. of difficulties.
To test the significance of $\mathrm{W}$, Chi-square test was applied using the following formula

$$
X^{2}=k(n-1) W \text { at (n-1) df. }
$$

\section{Mann-Whitney U-test}

For analyzing the constraints felt by the beneficiaries of the scheme, this technique was used.

The identified constraints were grouped under 14 sub headings and enquired whether they agree or disagree with the statement. For each constraint, two groups of beneficiaries were identified i.e., agreed and disagreed beneficiaries. The tool was employed to find out whether there was significant agreement among all the respondents.

' $U$ ' value was computed with the help of the following formula.

$\mathrm{U}=\mathrm{n}_{1} \cdot \mathrm{n}_{2}+\frac{\mathrm{n}_{1}\left(\mathrm{n}_{1}+1\right)}{2}$

Where,

$\mathrm{n}_{1}=$ Number of cases in first group

$\mathrm{n}_{2}=$ Number of cases in second group

$\mathrm{R}_{1}=$ Sum of the ranks assigned to group whose sample size is $n_{i}$

The significance of ' $U$ ' was tested with the help of table of probabilities associated with observed values of ' $U$ ' in the Mann-Whitney test.

\section{Results and Discussion}

Opinions of beneficiaries on planning of the scheme

Individual beneficiaries of MGNREGS were subjected to different predesigned 
questionnaires (Table 2) to assess the opinion of beneficiaries on different logical parameters on the planning aspects of MGNREGS in both HEMs and LEMs of Karimnagar.

The results revealed that the present system of planning, participation of villagers in selection of work and village women choice in planning process in both HEMs and LEMs of Karimnagar appears to be very much as majority of sample units have agreed to this opinion besides agreeing to the works utility under MGNREGS to the society.

On subjecting the data to the Mean Standard Deviation Technique Analysis, three levels of agreement of the opinions of respondents was observed. They were shown in Table 3.

Thus, it can be concluded that women have major role in MGNREGS planning along with men in the village.

It was confirmed that 85 per cent has agreed to the positive side of the opinions.

Similar results were noticed (Table 4) in Medak district in both HEMs and LEMs in confirming of the opinion of beneficiaries in planning of the programme with people's participation only (Table 5).

\section{Opinions of beneficiaries implementation of the scheme}

Beneficiaries' opinion on implementation of the programme has been obtained and presented in Table 6.

The data indicates that the implementation of programme was highly positive in two aspects. Payments on equality basis without gender discrimination and more involvement of women force in implementation of the programme. Further analysis of the data indicated that 78 per cent of the sample units were in positive opinion towards implementation of MGNREGS.

The striking feature was that MGNREGS has made no gender discrimination in wage remunerations as is a practice prevails in rural India as women receives relatively less remuneration for their labour contribution.

The second aspect is that giving due share to women in works allotment has been followed in MGNREGS as per the mandate which states that 33 per cent of work should be provided to the women. Thus, it can be concluded explicitly that the MGNREGS has brought a radical social changes to mitigate the gender discrimination in wage remuneration which is one of the government's major effort in all the programmes implementation.

Similar results were noticed even in Medak district data (Table 8) where the beneficiary farmers expressed a positive indication on implementation of MGNREGS in both LEMs and HEMs (Table 9).

Table.1 Sample villages selection procedure

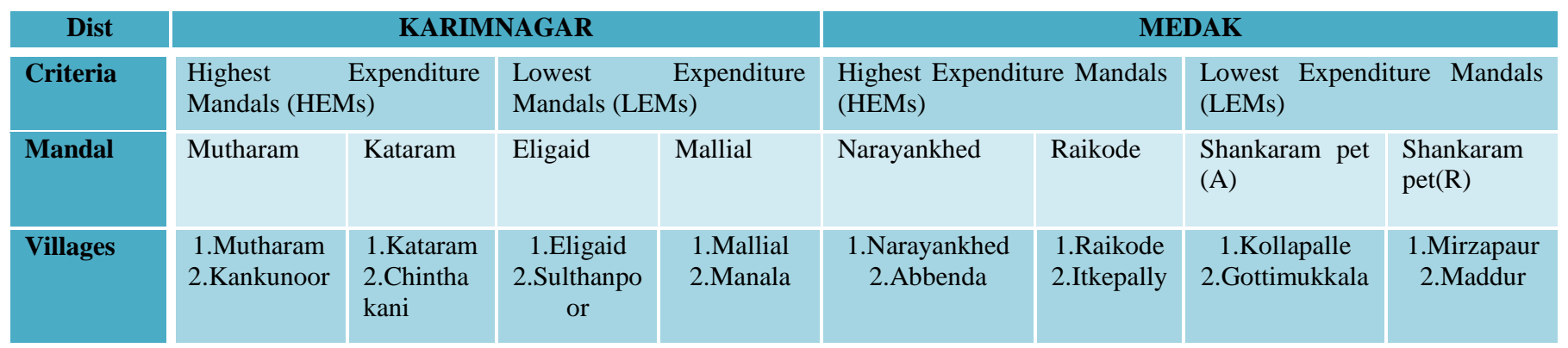


Table.2 MGNREGS beneficiaries' opinion on planning of the programme in Karimnagar district

\begin{tabular}{|c|c|c|c|c|c|c|c|c|c|c|c|}
\hline \multirow[t]{3}{*}{ S. No } & \multirow[t]{3}{*}{ Parameter } & \multicolumn{10}{|c|}{ Karimnagar } \\
\hline & & \multicolumn{5}{|c|}{ Highest expenditure mandals } & \multicolumn{5}{|c|}{ Lowest expenditure mandals } \\
\hline & & Excellent & $\begin{array}{l}\text { Very } \\
\text { good }\end{array}$ & Good & Moderate & Poor & $\begin{array}{c}\text { Excellen } \\
\mathbf{t}\end{array}$ & $\begin{array}{l}\text { Very } \\
\text { good }\end{array}$ & Good & Moderate & Poor \\
\hline 1 & Present system of planning & $\begin{array}{c}5 \\
(15.62)\end{array}$ & $\begin{array}{c}15 \\
(46.87)\end{array}$ & $\begin{array}{c}10 \\
(31.25)\end{array}$ & $\begin{array}{c}2 \\
(6.25)\end{array}$ & $\begin{array}{c}0 \\
(0)\end{array}$ & $\begin{array}{c}10 \\
(31.25)\end{array}$ & $\begin{array}{c}12 \\
(37.5)\end{array}$ & $\begin{array}{c}10 \\
(31.25)\end{array}$ & $\begin{array}{c}0 \\
(0)\end{array}$ & $\begin{array}{c}0 \\
(0)\end{array}$ \\
\hline 2 & $\begin{array}{l}\text { Present level of participation of villagers in the selection of } \\
\text { work }\end{array}$ & $\begin{array}{c}3 \\
(9.37)\end{array}$ & $\begin{array}{c}20 \\
(62.5)\end{array}$ & $\begin{array}{c}7 \\
(21.87)\end{array}$ & $\begin{array}{c}2 \\
(6.25)\end{array}$ & $\begin{array}{c}0 \\
(0)\end{array}$ & $\begin{array}{c}2 \\
(6.25)\end{array}$ & $\begin{array}{c}16 \\
(50)\end{array}$ & $\begin{array}{c}10 \\
(31.25)\end{array}$ & $\begin{array}{c}2 \\
(6.25)\end{array}$ & $\begin{array}{c}2 \\
(6.25)\end{array}$ \\
\hline 3 & Role of villagers in selection of the work site & $\begin{array}{c}6 \\
(18.75)\end{array}$ & $\begin{array}{c}14 \\
(43.75)\end{array}$ & $\begin{array}{c}8 \\
(25)\end{array}$ & $\begin{array}{c}4 \\
(12.5)\end{array}$ & $\begin{array}{c}0 \\
(0)\end{array}$ & $\begin{array}{c}6 \\
(18.75)\end{array}$ & $\begin{array}{c}9 \\
(28.12)\end{array}$ & $\begin{array}{c}8 \\
(25)\end{array}$ & $\begin{array}{c}8 \\
(25)\end{array}$ & $\begin{array}{c}1 \\
(3.12)\end{array}$ \\
\hline 4 & $\begin{array}{l}\text { Influence of contractors and influential people in selection of } \\
\text { works and work sites }\end{array}$ & $\begin{array}{c}0 \\
(0)\end{array}$ & $\begin{array}{c}4 \\
(12.5)\end{array}$ & $\begin{array}{c}5 \\
(15.62)\end{array}$ & $\begin{array}{c}8 \\
(25)\end{array}$ & $\begin{array}{c}15 \\
(46.87)\end{array}$ & $\begin{array}{c}0 \\
(0)\end{array}$ & $\begin{array}{l}2 \\
(6.25)\end{array}$ & $5(15.62)$ & $\begin{array}{c}8 \\
(25)\end{array}$ & $\begin{array}{c}17 \\
(53.12)\end{array}$ \\
\hline 5 & Share of village women in planning process & $\begin{array}{c}12 \\
(37.5)\end{array}$ & $\begin{array}{c}10 \\
(31.25)\end{array}$ & $\begin{array}{c}9 \\
(28.12)\end{array}$ & $\begin{array}{c}1 \\
(3.12)\end{array}$ & $\begin{array}{c}0 \\
(0)\end{array}$ & $\begin{array}{c}6 \\
(18.75)\end{array}$ & $\begin{array}{c}12 \\
(37.5)\end{array}$ & $\begin{array}{c}10 \\
(31.25)\end{array}$ & $\begin{array}{c}3 \\
(9.37)\end{array}$ & $\begin{array}{c}1 \\
(3.12)\end{array}$ \\
\hline$\overline{6}$ & $\begin{array}{l}\text { Planning is going on such a way that it is not creating labour } \\
\text { shortage to farming especially in peak periods }\end{array}$ & $\begin{array}{c}2 \\
(6.25)\end{array}$ & $\begin{array}{c}8 \\
(25)\end{array}$ & $\begin{array}{c}8 \\
(25)\end{array}$ & $\begin{array}{c}3 \\
(9.37)\end{array}$ & $\begin{array}{c}11 \\
(34.37)\end{array}$ & $\begin{array}{c}1 \\
(3.12)\end{array}$ & $\begin{array}{c}13 \\
(40.62)\end{array}$ & $\begin{array}{c}8 \\
(25)\end{array}$ & $\begin{array}{c}7 \\
(21.87)\end{array}$ & $\begin{array}{c}3 \\
(9.37)\end{array}$ \\
\hline 7 & MGNREGS staff involvement in planning & $\begin{array}{c}5 \\
(15.62)\end{array}$ & $\begin{array}{c}11 \\
(34.37)\end{array}$ & $\begin{array}{c}14 \\
(43.75)\end{array}$ & $\begin{array}{c}2 \\
(6.25)\end{array}$ & $\begin{array}{c}0 \\
(0)\end{array}$ & $\begin{array}{c}1 \\
(3.12)\end{array}$ & $\begin{array}{c}16 \\
(50)\end{array}$ & $12(37.5)$ & $\begin{array}{c}2 \\
(6.25)\end{array}$ & $\begin{array}{c}1 \\
(3.12)\end{array}$ \\
\hline 8 & Selection of work utility to society & $\begin{array}{c}3 \\
(9.37)\end{array}$ & $\begin{array}{c}9 \\
(28.12)\end{array}$ & $\begin{array}{c}11 \\
34.37)\end{array}$ & $\begin{array}{c}7 \\
(21.87)\end{array}$ & $\begin{array}{c}2 \\
(6.25)\end{array}$ & $\begin{array}{c}3 \\
(9.37)\end{array}$ & $\begin{array}{c}12 \\
(37.5)\end{array}$ & $\begin{array}{c}8 \\
(25)\end{array}$ & $\begin{array}{c}5 \\
(15.62)\end{array}$ & $\begin{array}{c}4 \\
(12.5)\end{array}$ \\
\hline
\end{tabular}

Note: Figures in parenthesis indicates percentage to the total.

Table.3 Mean standard deviation technique analysis for Karimnagar district

\begin{tabular}{|c|c|c|c|c|c|c|c|}
\hline \multirow[t]{2}{*}{ S. No } & \multirow[t]{2}{*}{ Agreement level } & \multicolumn{3}{|c|}{ Highest expenditure mandals } & \multicolumn{3}{|c|}{ Lowest expenditure mandals } \\
\hline & & Range & $\begin{array}{l}\text { Frequency } \\
\text { (n) }\end{array}$ & Percent & Range & $\begin{array}{l}\text { Frequency } \\
\text { (n) }\end{array}$ & Percent \\
\hline 1 & $\begin{array}{l}\text { Lower agreement } \\
\text { (< Mean - SD) }\end{array}$ & $<1.291937$ & 7 & 17.5 & $<1.470757$ & 8 & 20 \\
\hline 2 & $\begin{array}{l}\text { Medium agreement } \\
\text { (= Mean +/- SD) }\end{array}$ & $\begin{array}{l}1.291937- \\
11.50806\end{array}$ & 27 & 67.5 & $\begin{array}{c}1.470757- \\
11.32924\end{array}$ & 24 & 60 \\
\hline 3 & $\begin{array}{l}\text { Higher agreement } \\
\text { (= Mean +SD) }\end{array}$ & $>11.50806$ & 6 & 15 & $>11.32924$ & 8 & 20 \\
\hline
\end{tabular}


Table.4 MGNREGS beneficiaries' opinion on Planning of the programme in Medak district

\begin{tabular}{|c|c|c|c|c|c|c|c|c|c|c|c|}
\hline \multirow{3}{*}{ S.No } & \multirow{3}{*}{ Parameter } & \multicolumn{10}{|c|}{ Medak } \\
\hline & & \multicolumn{5}{|c|}{ Highest expenditure mandals } & \multicolumn{5}{|c|}{ Lowest expenditure mandals } \\
\hline & & Excellent & $\begin{array}{l}\text { Very } \\
\text { good }\end{array}$ & Good & Moderate & Poor & Excellent & $\begin{array}{l}\text { Very } \\
\text { good }\end{array}$ & Good & Moderate & Poor \\
\hline 1 & Present system of planning & $\begin{array}{c}3 \\
(9.37)\end{array}$ & $\begin{array}{c}12 \\
(37.5)\end{array}$ & $\begin{array}{c}13 \\
(40.62)\end{array}$ & $\begin{array}{c}4 \\
(12.5)\end{array}$ & $\begin{array}{c}0 \\
(0)\end{array}$ & $\begin{array}{c}4 \\
(12.5)\end{array}$ & $\begin{array}{c}17 \\
(53.12)\end{array}$ & $9(28.12)$ & $\begin{array}{c}2 \\
(6.25)\end{array}$ & $\begin{array}{c}0 \\
(0)\end{array}$ \\
\hline 2 & Present level of participation of villagers in the selection of work & $\begin{array}{c}3 \\
(9.37)\end{array}$ & $\begin{array}{c}10 \\
(31.25)\end{array}$ & $\begin{array}{c}10 \\
(31.25)\end{array}$ & $\begin{array}{c}6 \\
(18.75)\end{array}$ & $\begin{array}{c}3 \\
(9.37)\end{array}$ & $\begin{array}{c}1 \\
(3.12)\end{array}$ & $\begin{array}{c}11 \\
(34.37)\end{array}$ & $17(53.12)$ & $\begin{array}{c}3 \\
(9.375)\end{array}$ & $\begin{array}{c}0 \\
(0)\end{array}$ \\
\hline 3 & Role of villagers in selection of the work site & $\begin{array}{c}1 \\
(3.12)\end{array}$ & $\begin{array}{c}9 \\
(28.12)\end{array}$ & $\begin{array}{c}10 \\
(31.25)\end{array}$ & $\begin{array}{c}7 \\
(21.87)\end{array}$ & $5(15.62)$ & $\begin{array}{c}2 \\
(6.25)\end{array}$ & $\begin{array}{c}9 \\
(28.12)\end{array}$ & $11(34.37)$ & $\begin{array}{c}9 \\
(28.12)\end{array}$ & $\begin{array}{c}1 \\
(3.12)\end{array}$ \\
\hline 4 & $\begin{array}{l}\text { Influence of contractors and influential people in selection of works } \\
\text { and work sites }\end{array}$ & $\begin{array}{c}0 \\
(0)\end{array}$ & $\begin{array}{c}1 \\
(3.12)\end{array}$ & $\begin{array}{c}3 \\
(9.37)\end{array}$ & $\begin{array}{c}5 \\
(15.62)\end{array}$ & $23(71.87)$ & $\begin{array}{c}0 \\
(0)\end{array}$ & $\begin{array}{c}0 \\
(0)\end{array}$ & $\begin{array}{c}4 \\
(12.5)\end{array}$ & $\begin{array}{c}16 \\
(50)\end{array}$ & $\begin{array}{c}12 \\
(37.5)\end{array}$ \\
\hline 5 & Share of village women in planning process & $\begin{array}{c}5 \\
(15.62)\end{array}$ & $\begin{array}{c}11 \\
(34.37)\end{array}$ & $\begin{array}{c}8 \\
(25)\end{array}$ & $\begin{array}{c}5 \\
(15.62)\end{array}$ & $\begin{array}{c}3 \\
(9.37)\end{array}$ & $\begin{array}{c}3 \\
(9.37)\end{array}$ & $\begin{array}{c}15 \\
(46.87)\end{array}$ & $\begin{array}{c}8 \\
(25)\end{array}$ & $\begin{array}{c}6 \\
(18.75)\end{array}$ & $\begin{array}{c}0 \\
(0)\end{array}$ \\
\hline 6 & $\begin{array}{l}\text { Planning is going on such a way that it is not creating labour shortage } \\
\text { to farming especially in peak periods }\end{array}$ & $\begin{array}{c}1 \\
(3.12)\end{array}$ & $\begin{array}{c}4 \\
(12.5)\end{array}$ & $\begin{array}{c}10 \\
(31.25)\end{array}$ & $\begin{array}{c}8 \\
(25)\end{array}$ & $9(28.12)$ & $\begin{array}{c}1 \\
(3.12)\end{array}$ & $\begin{array}{c}4 \\
(12.5)\end{array}$ & $9(28.12)$ & $\begin{array}{c}10 \\
(31.25)\end{array}$ & $\begin{array}{c}8 \\
(25)\end{array}$ \\
\hline 7 & MGNREGS staff involvement in planning & $\begin{array}{c}3 \\
(9.37)\end{array}$ & $\begin{array}{c}10 \\
(31.25)\end{array}$ & $\begin{array}{c}10 \\
(31.25)\end{array}$ & $\begin{array}{c}8 \\
(25)\end{array}$ & $\begin{array}{c}1 \\
(3.12)\end{array}$ & $\begin{array}{c}1 \\
(3.12)\end{array}$ & $\begin{array}{c}11 \\
(34.37)\end{array}$ & $11(34.37)$ & $\begin{array}{c}8 \\
(25)\end{array}$ & $\begin{array}{c}1 \\
(3.12)\end{array}$ \\
\hline 8 & Selection of work utility to society & $\begin{array}{c}2 \\
(6.25)\end{array}$ & $\begin{array}{c}15 \\
(46.87)\end{array}$ & $\begin{array}{c}9 \\
(28.12)\end{array}$ & $\begin{array}{c}6 \\
(18.75)\end{array}$ & $\begin{array}{c}0 \\
(0)\end{array}$ & $\begin{array}{c}1 \\
(3.12)\end{array}$ & $\begin{array}{c}9 \\
(28.12)\end{array}$ & $11(34.37)$ & $\begin{array}{c}8 \\
(25)\end{array}$ & $\begin{array}{c}3 \\
(9.37)\end{array}$ \\
\hline
\end{tabular}

Note: Figures in parenthesis indicates percentage to the total.

Table.5 MGNREGS beneficiaries' opinion on Implementation of the programme in Karimnagar district

\begin{tabular}{|c|c|c|c|c|c|c|c|c|c|c|c|}
\hline \multirow[b]{3}{*}{ S.No } & \multirow[b]{3}{*}{ Parameter } & \multicolumn{10}{|c|}{ Karimnagar } \\
\hline & & \multicolumn{5}{|c|}{ Highest expenditure mandals } & \multicolumn{5}{|c|}{ Lowest expenditure mandals } \\
\hline & & $\begin{array}{l}\text { Very } \\
\text { high }\end{array}$ & High & Medium & Low & Very low & Very high & High & Medium & Low & Very low \\
\hline 1 & Delays in wage payments & $\begin{array}{c}2 \\
(6.25)\end{array}$ & $\begin{array}{c}2 \\
(6.25)\end{array}$ & $\begin{array}{c}9 \\
(28.12)\end{array}$ & $\begin{array}{c}3 \\
(9.37)\end{array}$ & $\begin{array}{c}16 \\
(50)\end{array}$ & $\begin{array}{c}3 \\
(9.37)\end{array}$ & $\begin{array}{c}4 \\
(12.5)\end{array}$ & $\begin{array}{c}5 \\
(15.62)\end{array}$ & $6(18.75)$ & $\begin{array}{c}14 \\
(43.75)\end{array}$ \\
\hline 2 & Inefficiency in work measurement & $\begin{array}{c}0 \\
(0)\end{array}$ & $\begin{array}{c}4 \\
(12.5)\end{array}$ & $\begin{array}{c}5 \\
(15.62)\end{array}$ & $\begin{array}{c}8 \\
(25)\end{array}$ & $\begin{array}{c}15 \\
(46.87)\end{array}$ & $\begin{array}{c}0 \\
(0)\end{array}$ & $\begin{array}{c}5 \\
(15.62)\end{array}$ & $\begin{array}{c}8 \\
(25)\end{array}$ & $5(15.62)$ & $\begin{array}{c}14 \\
(43.75)\end{array}$ \\
\hline 3 & Manipulation of muster rolls & $\begin{array}{c}1 \\
(3.12)\end{array}$ & $\begin{array}{c}0 \\
(0)\end{array}$ & $\begin{array}{c}9 \\
(28.12)\end{array}$ & $9(28.12)$ & $\begin{array}{c}13 \\
(40.62)\end{array}$ & $\begin{array}{c}0 \\
(0)\end{array}$ & $\begin{array}{c}6 \\
(18.75)\end{array}$ & $\begin{array}{c}6 \\
(18.75)\end{array}$ & $7(21.87)$ & $\begin{array}{c}13 \\
(40.62)\end{array}$ \\
\hline 4 & Allotment of $33 \%$ for women & $\begin{array}{c}11 \\
(34.37)\end{array}$ & $\begin{array}{c}14 \\
(43.75)\end{array}$ & $\begin{array}{c}7 \\
\text { (21.87) }\end{array}$ & $\begin{array}{c}0 \\
(0)\end{array}$ & $\begin{array}{c}0 \\
(0)\end{array}$ & $\begin{array}{c}3 \\
(9.37)\end{array}$ & $\begin{array}{c}7 \\
\text { (21.87) }\end{array}$ & $\begin{array}{c}11 \\
(34.37)\end{array}$ & $9(28.12)$ & $\begin{array}{c}2 \\
(6.25)\end{array}$ \\
\hline 5 & Equal payments to male and female & $\begin{array}{c}21 \\
(65.62)\end{array}$ & $\begin{array}{c}5 \\
(15.62)\end{array}$ & $\begin{array}{c}4 \\
(12.5)\end{array}$ & $\begin{array}{c}2 \\
(6.25)\end{array}$ & $\begin{array}{c}0 \\
(0)\end{array}$ & $19(59.37)$ & $\begin{array}{c}6 \\
(18.75)\end{array}$ & $\begin{array}{c}5 \\
(15.62)\end{array}$ & $\begin{array}{c}1 \\
(3.12)\end{array}$ & $\begin{array}{c}1 \\
(3.12)\end{array}$ \\
\hline 6 & Unhappy with the frequency of payment of wages & $\begin{array}{c}0 \\
(0)\end{array}$ & $\begin{array}{c}3 \\
(9.37)\end{array}$ & $\begin{array}{c}7 \\
\text { (21.87) }\end{array}$ & $9(28.12)$ & $\begin{array}{c}13 \\
(40.62)\end{array}$ & $\begin{array}{c}0 \\
(0)\end{array}$ & $\begin{array}{c}2 \\
(6.25)\end{array}$ & $\begin{array}{c}11 \\
(34.37)\end{array}$ & $9(28.12)$ & $\begin{array}{c}10 \\
(31.25)\end{array}$ \\
\hline 7 & Staff dedication in implementation & $5(15.62)$ & $\begin{array}{c}11 \\
(34.37)\end{array}$ & $\begin{array}{c}13 \\
(40.62)\end{array}$ & $\begin{array}{c}3 \\
(9.37)\end{array}$ & $\begin{array}{c}0 \\
(0)\end{array}$ & $5(15.62)$ & $\begin{array}{c}8 \\
(25)\end{array}$ & $\begin{array}{c}11 \\
(34.37)\end{array}$ & $6(18.75)$ & $\begin{array}{c}2 \\
(6.25)\end{array}$ \\
\hline 8 & Effect of social auditing & $\begin{array}{c}10 \\
(31.25)\end{array}$ & $\begin{array}{c}9 \\
(28.12)\end{array}$ & $\begin{array}{c}9 \\
(28.12)\end{array}$ & $\begin{array}{c}4 \\
(12.5)\end{array}$ & $\begin{array}{c}0 \\
(0)\end{array}$ & $9(28.12)$ & $\begin{array}{c}9 \\
(28.12)\end{array}$ & $\begin{array}{c}10 \\
(31.25)\end{array}$ & $\begin{array}{c}3 \\
(9.37)\end{array}$ & $\begin{array}{c}1 \\
(3.12)\end{array}$ \\
\hline
\end{tabular}

Note: Figures in parenthesis indicates percentage to the total. 
Table.6 Mean standard deviation technique analysis for Medak district

\begin{tabular}{|c|c|c|c|c|c|c|c|}
\hline \multirow{2}{*}{$\begin{array}{l}\text { S. } \\
\text { No }\end{array}$} & \multirow[t]{2}{*}{ Agreement level } & \multicolumn{3}{|c|}{ Highest expenditure area } & \multicolumn{3}{|c|}{ Lowest expenditure area } \\
\hline & & Range & $\begin{array}{l}\text { Frequency } \\
\text { (n) }\end{array}$ & Percent & Range & $\begin{array}{l}\text { Frequency } \\
\text { (n) }\end{array}$ & Percent \\
\hline 1 & $\begin{array}{l}\text { Lower agreement } \\
(<\text { Mean - SD) }\end{array}$ & $<1.597223$ & 7 & 17.5 & $<1.197437$ & 11 & 27.5 \\
\hline 2 & $\begin{array}{l}\text { Medium agreement } \\
\text { (= Mean +/- SD) }\end{array}$ & $\begin{array}{c}1.597223- \\
11.20278\end{array}$ & 29 & 72.5 & $\begin{array}{c}1.197437- \\
11.60256\end{array}$ & 24 & 60 \\
\hline 3 & $\begin{array}{l}\text { Higher agreement } \\
(=\text { Mean +SD) }\end{array}$ & $>11.20278$ & 4 & 10 & $>11$ & 5 & 12.5 \\
\hline
\end{tabular}

Table.7 Analysis results of opinions of beneficiaries of Karimnagar on implementation

\begin{tabular}{|c|c|c|c|c|c|c|c|c|c|}
\hline \multicolumn{5}{|c|}{ Highest expenditure mandals } & \multicolumn{5}{|c|}{ Lowest expenditure mandals } \\
\hline S. No & Opinion & Class & $\begin{array}{c}\text { Frequency } \\
\text { (n) }\end{array}$ & Percent & S. No & Opinion & Class & $\begin{array}{c}\text { Frequenc } \\
\text { y (n) }\end{array}$ & Percent \\
\hline 1 & Very good & $0-21$ & 22 & 55 & 1 & Very good & $0-19$ & 20 & 50 \\
\hline 2 & Good & $22-42$ & 9 & 22.5 & 2 & Good & $20-38$ & 14 & 35 \\
\hline 3 & Moderate & $43-63$ & 4 & 10 & 3 & Moderate & $39-57$ & 2 & 5 \\
\hline 4 & Bad & $64-84$ & 4 & 10 & 4 & $\mathrm{Bad}$ & $58-76$ & 3 & 7.5 \\
\hline 5 & Very bad & $85-105$ & 1 & 2.5 & 5 & Very bad & $77-95$ & 1 & 2.5 \\
\hline & \multicolumn{2}{|c|}{ Total } & 40 & 100 & & \multicolumn{2}{|c|}{ Total } & 40 & 100 \\
\hline
\end{tabular}

Table.8 MGNREGS beneficiaries' opinion on Implementation of the programme in Medak district

\begin{tabular}{|c|c|c|c|c|c|c|c|c|c|c|}
\hline \multirow{3}{*}{ Parameter } & \multicolumn{10}{|c|}{ Medak } \\
\hline & \multicolumn{5}{|c|}{ Highest expenditure mandals } & \multicolumn{5}{|c|}{ Lowest expenditure mandals } \\
\hline & $\begin{array}{l}\text { Very } \\
\text { high }\end{array}$ & High & Medium & Low & Very low & Very high & High & Medium & Low & Very low \\
\hline Delays in wage payments & $\begin{array}{c}2 \\
(6.25)\end{array}$ & $\begin{array}{c}4 \\
(12.5)\end{array}$ & $\begin{array}{c}9 \\
(28.12)\end{array}$ & $\begin{array}{c}8 \\
(25)\end{array}$ & $9(28.12)$ & $\begin{array}{c}0 \\
(0)\end{array}$ & $\begin{array}{c}12 \\
(37.5)\end{array}$ & $\begin{array}{c}8 \\
(25)\end{array}$ & $\begin{array}{c}10 \\
(31.25)\end{array}$ & $\begin{array}{c}2 \\
(6.25)\end{array}$ \\
\hline Inefficiency in work measurement & $\begin{array}{c}0 \\
(0)\end{array}$ & $\begin{array}{c}3 \\
(9.37)\end{array}$ & $\begin{array}{c}4 \\
(12.5)\end{array}$ & $\begin{array}{c}15 \\
(46.87)\end{array}$ & $\begin{array}{c}10 \\
(31.25)\end{array}$ & $\begin{array}{c}0 \\
(0)\end{array}$ & $\begin{array}{c}4 \\
(12.5)\end{array}$ & $\begin{array}{c}8 \\
(25)\end{array}$ & $\begin{array}{c}14 \\
(43.75)\end{array}$ & $\begin{array}{c}6 \\
(18.75)\end{array}$ \\
\hline Manipulation of muster rolls & $\begin{array}{c}2 \\
(6.25)\end{array}$ & $\begin{array}{c}3 \\
(9.37)\end{array}$ & $\begin{array}{c}10 \\
(31.25)\end{array}$ & $\begin{array}{c}6 \\
(18.75)\end{array}$ & $\begin{array}{c}11 \\
(34.37)\end{array}$ & $\begin{array}{c}0 \\
(0)\end{array}$ & $\begin{array}{c}3 \\
(9.37)\end{array}$ & $\begin{array}{c}4 \\
(12.5)\end{array}$ & $\begin{array}{c}12 \\
(37.5)\end{array}$ & $\begin{array}{c}13 \\
(40.62)\end{array}$ \\
\hline Allotment of $33 \%$ for women & $6(18.75)$ & $\begin{array}{c}17 \\
(53.12)\end{array}$ & $\begin{array}{c}6 \\
(18.75)\end{array}$ & $\begin{array}{c}1 \\
(3.12)\end{array}$ & $\begin{array}{c}2 \\
(6.25)\end{array}$ & $13(40.62)$ & $\begin{array}{c}14 \\
(43.75)\end{array}$ & $\begin{array}{c}5 \\
(15.62)\end{array}$ & $\begin{array}{c}0 \\
(0)\end{array}$ & $\begin{array}{c}0 \\
(0)\end{array}$ \\
\hline Equal payments to male and female & $12(37.5)$ & $\begin{array}{c}15 \\
(46.87)\end{array}$ & $\begin{array}{c}4 \\
(12.5)\end{array}$ & $\begin{array}{c}1 \\
(3.12)\end{array}$ & $\begin{array}{c}0 \\
(0)\end{array}$ & $\begin{array}{c}24 \\
(75)\end{array}$ & $\begin{array}{c}8 \\
(25)\end{array}$ & $\begin{array}{c}0 \\
(0)\end{array}$ & $\begin{array}{c}0 \\
(0)\end{array}$ & $\begin{array}{c}0 \\
(0)\end{array}$ \\
\hline Unhappy with the frequency of payment of wages & $\begin{array}{c}2 \\
(6.25)\end{array}$ & $\begin{array}{c}4 \\
(12.5)\end{array}$ & $\begin{array}{c}11 \\
(34.37)\end{array}$ & $\begin{array}{c}5 \\
(15.62)\end{array}$ & $\begin{array}{c}10 \\
(31.25)\end{array}$ & $\begin{array}{c}0 \\
(0)\end{array}$ & $\begin{array}{c}7 \\
(21.87)\end{array}$ & $\begin{array}{c}7 \\
(21.87)\end{array}$ & $\begin{array}{c}13 \\
(40.62)\end{array}$ & $\begin{array}{c}5 \\
(15.62)\end{array}$ \\
\hline Staff dedication in implementation & $\begin{array}{c}1 \\
(3.12)\end{array}$ & $\begin{array}{c}7 \\
(21.87)\end{array}$ & $\begin{array}{c}14 \\
(43.75)\end{array}$ & $\begin{array}{c}7 \\
(21.87)\end{array}$ & $\begin{array}{c}3 \\
(9.37)\end{array}$ & $\begin{array}{c}6 \\
(18.75)\end{array}$ & $\begin{array}{c}10 \\
(31.25)\end{array}$ & $\begin{array}{c}11 \\
(34.37)\end{array}$ & $\begin{array}{c}3 \\
(9.37)\end{array}$ & $\begin{array}{c}2 \\
(6.25)\end{array}$ \\
\hline Effect of social auditing & $7(21.87)$ & $\begin{array}{c}16 \\
(50)\end{array}$ & $\begin{array}{c}8 \\
(25)\end{array}$ & $\begin{array}{c}1 \\
(3.12)\end{array}$ & $\begin{array}{c}0 \\
(0)\end{array}$ & $\begin{array}{c}12 \\
(37.5)\end{array}$ & $\begin{array}{c}12 \\
(37.5)\end{array}$ & $\begin{array}{c}5 \\
(15.62)\end{array}$ & $\begin{array}{c}2 \\
(6.25)\end{array}$ & $\begin{array}{c}1 \\
(3.12)\end{array}$ \\
\hline
\end{tabular}

Note: Figures in parenthesis indicates percentage to the total 
Table.9 Analysis results of opinions of beneficiaries of Medak on implementation

\begin{tabular}{|c|c|c|c|c|c|c|c|c|c|}
\hline \multicolumn{5}{|c|}{ Highest expenditure mandals } & \multicolumn{5}{|c|}{ Lowest expenditure mandals } \\
\hline S. No & Opinion & Class & $\begin{array}{c}\text { Frequenc } \\
\mathbf{y}(\mathbf{n})\end{array}$ & Percent & S. No & Opinion & Class & $\begin{array}{c}\text { Frequenc } \\
\text { y (n) }\end{array}$ & Percent \\
\hline 1 & Very good & $0-13.6$ & 18 & 45 & 1 & Very good & $0-24$ & 24 & 60 \\
\hline 2 & Good & $13.7-27.2$ & 6 & 15 & 2 & Good & $25-48$ & 9 & 22.5 \\
\hline 3 & Moderate & $27.3-40.8$ & 6 & 15 & 3 & Moderate & $49-72$ & 6 & 15 \\
\hline 4 & Bad & $40.9-54.4$ & 4 & 10 & 4 & Bad & $73-96$ & 0 & 0 \\
\hline 5 & Very bad & $54.5-68$ & 6 & 15 & 5 & Very bad & $97-120$ & 1 & 2.5 \\
\hline & \multicolumn{2}{|c|}{ Total } & 40 & 100 & & \multicolumn{2}{|c|}{ Total } & 40 & 100 \\
\hline
\end{tabular}

Table.10 Opinions of MGNREGS implementing agencies of the scheme

\begin{tabular}{|l|l|c|c|c|c|c|}
\hline \multicolumn{1}{|c|}{ Parameter } & \multicolumn{1}{|c|}{ Very } & High & Medium & Low & Very \\
low
\end{tabular}

Note: Figures in parenthesis indicates percentage to the total.

Table.11 Analysis results of opinion of implementing agencies

\begin{tabular}{|c|l|c|c|c|}
\hline S. No & \multicolumn{1}{|c|}{ Opinion } & Class & $\begin{array}{c}\text { Frequency } \\
(\mathbf{n})\end{array}$ & $\begin{array}{c}\text { Percentage } \\
(\%)\end{array}$ \\
\hline $\mathbf{1}$ & Very good & $0-21$ & 31 & 77.5 \\
2 & Good & $22-43$ & 3 & 7.5 \\
3 & Moderate & $44-65$ & 2 & 5.0 \\
4 & Bad & $66-87$ & 2 & 5.0 \\
5 & Very bad & $88-109$ & 2 & 5.0 \\
\hline & & Total & $\mathbf{4 0}$ & $\mathbf{1 0 0}$ \\
\hline
\end{tabular}


Table.12 Allotment of ranks to each procedural difficulty by implementing agency

\begin{tabular}{|c|c|c|c|c|c|c|c|c|c|c|}
\hline \multirow{2}{*}{$\begin{array}{l}\text { Implementing } \\
\text { agency }\end{array}$} & \multicolumn{10}{|c|}{ Ranks } \\
\hline & 1 & 2 & 3 & 4 & 5 & 6 & 7 & 8 & 9 & 10 \\
\hline 1 & 2 & 4 & 5 & 7 & 3 & 2 & 2 & 3 & 3 & 6 \\
\hline 2 & 3 & 2 & 7 & 4 & 4 & 3 & 3 & 6 & 5 & 6 \\
\hline 3 & 1 & 1 & 3 & 5 & 4 & 6 & 2 & 6 & 2 & 3 \\
\hline 4 & 4 & 4 & 8 & 5 & 6 & 5 & 1 & 4 & 2 & 8 \\
\hline 5 & 2 & 2 & 5 & 4 & 3 & 1 & 4 & 6 & 3 & 8 \\
\hline 6 & 2 & 2 & 5 & 4 & 6 & 4 & 3 & 4 & 2 & 7 \\
\hline 7 & 1 & 2 & 6 & 5 & 2 & 1 & 3 & 6 & 3 & 8 \\
\hline 8 & 2 & 2 & 4 & 3 & 3 & 2 & 2 & 5 & 3 & 6 \\
\hline 9 & 1 & 2 & 6 & 5 & 6 & 4 & 5 & 3 & 6 & 5 \\
\hline 10 & 1 & 3 & 7 & 2 & 6 & 7 & 3 & 2 & 3 & 4 \\
\hline 11 & 2 & 3 & 4 & 6 & 5 & 2 & 5 & 6 & 4 & 6 \\
\hline 12 & 2 & 1 & 3 & 4 & 5 & 3 & 4 & 6 & 4 & 7 \\
\hline 13 & 1 & 4 & 6 & 6 & 6 & 3 & 3 & 5 & 5 & 8 \\
\hline 14 & 2 & 1 & 4 & 3 & 3 & 1 & 6 & 6 & 3 & 5 \\
\hline 15 & 1 & 1 & 3 & 4 & 6 & 3 & 6 & 7 & 8 & 8 \\
\hline 16 & 1 & 3 & 5 & 5 & 5 & 4 & 4 & 5 & 5 & 6 \\
\hline 17 & 3 & 3 & 4 & 4 & 3 & 3 & 3 & 5 & 3 & 5 \\
\hline 18 & 2 & 1 & 2 & 2 & 6 & 2 & 2 & 7 & 2 & 6 \\
\hline 19 & 1 & 2 & 4 & 3 & 7 & 4 & 3 & 3 & 5 & 4 \\
\hline 20 & 2 & 2 & 4 & 2 & 3 & 3 & 4 & 5 & 3 & 3 \\
\hline 21 & 3 & 2 & 2 & 5 & 6 & 3 & 7 & 4 & 7 & 8 \\
\hline 22 & 2 & 3 & 4 & 2 & 3 & 4 & 2 & 3 & 7 & 4 \\
\hline 23 & 2 & 4 & 1 & 6 & 4 & 3 & 2 & 4 & 6 & 4 \\
\hline 24 & 1 & 3 & 4 & 5 & 2 & 3 & 5 & 7 & 7 & 6 \\
\hline $\mathbf{R i}$ & 44 & 57 & 106 & 101 & 107 & 76 & 84 & 118 & 101 & 141 \\
\hline
\end{tabular}

Table.13 Agreement and disagreement pattern of the beneficiaries with the identified constraints

\begin{tabular}{|c|c|c|c|c|c|c|c|c|c|c|c|c|c|c|c|}
\hline Constraint & 1 & 2 & 3 & 4 & 5 & 6 & 7 & 8 & 9 & 10 & 11 & 12 & 13 & 14 & 15 \\
\hline $\begin{array}{l}\text { Agreed } \\
\text { respondents }\end{array}$ & 60 & 71 & 53 & 62 & 60 & 15 & 61 & 30 & 27 & 40 & 16 & 19 & 59 & 38 & 76 \\
\hline Percentage & 46.88 & 55.47 & 41.41 & 48.44 & 46.88 & 11.72 & 47.66 & 23.44 & 21.09 & 31.25 & 12.5 & 14.84 & 46.09 & 29.69 & 59.38 \\
\hline $\begin{array}{l}\text { Disagreed } \\
\text { respondents }\end{array}$ & 68 & 57 & 75 & 66 & 68 & 113 & 67 & 98 & 101 & 88 & 112 & 109 & 69 & 90 & 52 \\
\hline Percentage & 53.12 & 44.53 & 58.59 & 51.56 & 53.12 & 88.28 & 52.34 & 76.56 & 78.91 & 68.75 & 87.5 & 85.16 & 53.91 & 70.31 & 40.62 \\
\hline
\end{tabular}

Table.14 Assignment of the ranks to each constraint

\begin{tabular}{|c|c|c|c|c|c|c|c|c|c|c|c|c|c|c|c|}
\hline Constraint & 1 & 2 & 3 & 4 & 5 & 6 & 7 & 8 & 9 & 10 & 11 & 12 & 13 & 14 & 15 \\
\hline Agreed respondents & 60 & 71 & 53 & 62 & 60 & 15 & 61 & 30 & 27 & 40 & 16 & 19 & 59 & 38 & 76 \\
\hline Rank allotted & 12.5 & 21 & 9 & 15 & 12.5 & 1 & 14 & 5 & 4 & 7 & 2 & 3 & 11 & 6 & 23 \\
\hline Disagreed respondents & 68 & 57 & 75 & 66 & 68 & 113 & 67 & 98 & 101 & 88 & 112 & 109 & 69 & 90 & 52 \\
\hline Rank allotted & 18.5 & 10 & 22 & 16 & 18.5 & 30 & 17 & 26 & 27 & 24 & 29 & 28 & 20 & 25 & 8 \\
\hline
\end{tabular}

Drawing conclusions exclusively based on the opinions of beneficiaries alone would be incomplete without considering the opinions of its implementing agencies. Hence, were made to survey the agencies involved in implementing MGNREGS (Table 10) like 
Gram panchayats, field assistants, engineering consultants, representatives of DWMA etc. Totally 24 respondents representing various implementing agencies opinions were collected.

It was found that the implementing agencies opinions too were positive for the programme and highlighted that there was no gender discrimination in wage remuneration and women groups were given due share in allotment of works as per the mandate of the scheme.

Thus, a conclusion can be made that MGNREGS in all its spirits has brought a favorable socioeconomic change through the implementation of programme in rural India which has been supported by beneficiaries in planning and implementation and also by implementing agencies in implementation of MGNREGS.

Thus, MGNREGS has brought economic benefits which was seen in income generation and social benefits in mitigating the gender discrimination especially wage remunerations were achieved as per stipulated mandate with which MGNREGS was planned with people's participation and implemented in all its true spirits for the upliftment of target groups. Further analysis (Table 11) supported that 85 $\%$ were in accordance with the accepted positive side of opinions.

Procedural difficulties as viewed by the 'MGNREGS' implementing agencies

Procedural difficulties in implementation of MGNREGS are quite common due to sociopolitical environments in which the bureaucracy has to function in rural India.

During the visits and pretesting the procedural difficulties experienced by the identified 24 implementing agencies in study area have been obtained and enlisted for the purpose of assigning the ranks as per the opinion of the representatives of the implementing agencies.

Kendall's coefficient of concordance $(w)$ test was employed for the purpose of analysis (Table 12).

The procedural difficulties experienced by the representatives of implementing agencies have been enlisted and presented below.

Finding out the most appropriate work.

Finding out the sufficient amount of work to meet the needs of all applicants.

Arranging funds.

Elimination of fake / undeserved candidates from the enrolment.

Maintenance of stock or assets or tools and implements.

Preparation of action plan.

Verification of applications and issuing of job cards.

Facing the interference of politicians and other influential people.

Lack of knowledge and skills on the part of staff.

Fabrication of muster rolls.

$\sum \mathrm{Ri}=44+57+106+101+107+76+84$

$+118+101+141=935$

$\overline{\mathrm{R}}=935 / 10=93.5$

$\bar{R} \mathrm{i}-\mathrm{R}=-49.5,-36.5,12.5,7.5,13.5,-17.5$, $9.5,24.5,7.5,47.5$

$\bar{\Sigma}(\mathrm{Ri}-\mathrm{R})^{2}=7486.5$ 


$$
\mathrm{W}=\frac{\mathbf{1 2} \sum(\mathbf{R i}-\overline{\mathbf{R}})^{2}}{\mathrm{~K}^{2}\left(\mathrm{~N}^{3}-\mathrm{N}\right)}=\frac{12 \times 7486.5}{24^{2}\left(10^{3}-10\right)}=\frac{89838}{576 \mathrm{X} 990}
$$

$=0.15754419$

The $\mathrm{X}^{2}$ value was calculated to test the significance of the test.

$\mathrm{X}^{2}=\mathrm{k}(\mathrm{n}-1) \mathrm{w}$ at $(\mathrm{n}-1) \mathrm{df}=24(10-1) \mathrm{X}$ $0.15754419=34.02955$

$\mathrm{X}^{2}$ table value at $\alpha=0.05$ with $9 \mathrm{df}=16.92$

Since the calculated value of $X^{2}$ (34.02955) was greater than the table value of 16.92 at 9 df with $\alpha=0.05$ level, it was concluded that the value was significant and that all the interviewed agencies were in agreement in ranking the procedural difficulties in implementation of the programme.

The rankings given to the difficulties like finding out the most appropriate work, finding out of sufficient amount of work (Venkateswarlu and Rao, 2011) and preparation of action plans (Bannerjee, 2009) reveal that these were the major procedural difficulties in MGNREGS implementation and the fabrication of muster rolls (Dutta, 2009) by lower cadre employees seems to be the less felt difficulty. The analysis helps to conclude that the implementing agencies have to make efforts to reduce the identified procedural difficulties with due attention.

\section{Constraints in the implementation of the scheme}

Along with the procedural difficulties during MGNREGS implementation, constraints too accompany the programme which can be prevented in future course on their proper identification and quantification.

Political interference coupled with bureaucratic problems too does prevail as constraints for grounding the scheme in rural areas (Akhtar and Azeez, 2012).

Beneficiaries in the study area have been subjected to certain identified constraints that may be encountered in programme implementation which are presented below.

Poor maintenance of records.

Illiteracy of the beneficiaries.

Bureaucratic resistance.

Inadequate professional and administrative staff.

Lack of training or under training.

Unawareness about the scheme.

Insufficient funds for a particular task.

Presence of contractors.

Use of machinery instead of manual work.

Local disputes and election codes etc.

Lower wage rate or paying at out dated wage rate.

Absence of social audit which is mandatory biannually.

Absence of work site facilities.

Delayed payments.

Working for only SC/STs land development and denied to work on their own land.

The Mann-whitney $U$ test was employed to analyze the constraints by assigning scores with those agreed and disagreed groups (Table 13). 
Ranks were assigned to the scores of agreed and disagreed groups together in ascending order. The ranks were ranged from 1 to $\mathrm{N}$. where $\mathrm{N}=\mathrm{n} 1+\mathrm{n} 2$. Averages of tied ranks were assigned to tied observations.

$$
\begin{aligned}
& 1 \rightarrow \mathrm{N}=\mathrm{n} 1+\mathrm{n} 2 \\
& 1 \rightarrow 30=15+15 \\
& \mathrm{U}=\mathrm{n}_{1} \mathrm{n}_{2}+\underline{\mathrm{n}_{1}\left(\mathrm{n}_{1}+1\right)}-\mathrm{R}_{1} \\
& 2 \\
& \mathrm{R} 1=12.5+21+9+15+12.5+1+14+5+ \\
& 4+7+2+3+11+6+23=146 \\
& \mathrm{U}=15 \times 15+15(15+1) / 2-146 \\
& \mathrm{U}=199
\end{aligned}
$$

From the table 14, the probability value of ' $U$ ' is 0.399 which was more than $\alpha(=0.05)$, hence accepted the null hypothesis (Ho) in favour of $\mathrm{H} 1$.

Constraints studied were felt equally severe by both the groups of respondents in successful implementation of the programme and it was suggested that the initiation of any policy measure by the government to address these constraints is needed.

As per the opinion of beneficiaries in both Karimnagar and Medak districts, planning process in MGNREGS was very good, a majority of $34.37 \%$ of beneficiaries in HEMs of Karimnagar opined that the selection of work utility to society is good whereas in LEMs, 37.5\% felt the same whereas in Medak also, $46.87 \%$ in HEMs, $34.37 \%$ in LEMs agreed to the same opinion.

Regarding the implementation of the scheme, majority of respondents in Karimnagar were not happy with the frequency of payments and staff dedication in implementation was high according to respondents of HEMs and it was low according to respondents of LEMs where as in Medak, majority in HEMs and LEMs said that they were happy with the frequency of payments. Staff dedication in implementation was at middling level in HEMs and LEMs. Effect of social auditing was very high in HEMs and it was moderate in LEMs in both the districts.

Major difficulties encountered were finding out the most appropriate and sufficient amount of work, preparation of action plans, verification and issuing of job cards, elimination of fake and undeserved candidates etc. in both Karimnagar and Medak districts whereas illiteracy of the beneficiaries, inadequate professional and administrative staff were found to be the major constraints in successful implementation of the scheme. Hence, addressing the said difficulties and constraints is very much required for its successful implementation in future.

\section{References}

Ahirrao, J. 2012. A brief scanning of MGNREGA. Kurukshetra. 60(9): 3336.

Akthar, S.M.J and Azeez, N.P.A. 2012. Budgetary allocation and its utilization MGNREGS- a view point. Kurukshetra. 60(6): 19-22.

Banerjee, H. 2009. NREGA: a study in Andaman and Nicobar islands. Kurukshetra. 58(2): 23-26.

Bhatti, B. 2012. Aadhar enabled payments for NREGA workers. Economic and Political Weekly. 47(49): 16-19.

Dutta, S. 2009. NREGS in West Bengal: success and challenges. Kurukshetra. 58(2): 31-33.

Foster, A. 2012. Creating good employment opportunities for the rural sector. Asian Development Review. 29(1): 1-28.

Mahalingam, N. 2011. Mahatma Gandhi National Rural Employment Guarantee Scheme (MGNREGS) to create 
permanent assets. Kisan World. 60(2): 3-4.

Pani, N and Iyer, C.G. 2012. National Strategies and Local Realities: The Greenfield Approach and the MGNREGS in Karnataka. India Review. 11(1): 1-22.

Shah, D. 2011. Implementation of MGNREGS and livelihood security of rural households in Maharashtra: a region wise analysis. Agricultural
Economics Research Review. 24 (Conference number): 561.

Srivastava, N and Srivasatava, R. 2010. Women, Work and Employment outcomes in Rural India. Economic and Political Weekly. 45(28): 49-60.

Venkateswarlu, B and Rao, C.A.R. 2011. Rainfed agriculture - concerns, opportunities and strategies. Yojana. 55(1): 37-40.

www.nrega.nic.in

\section{How to cite this article:}

Kumara Swamy, D., C.V. Hanumanthaiah, P. Parthasarathy Rao, K. Suhasini, V.V. Narendranath and Vijaya Kumari, R. 2018. An Analysis of the Perspectives of 'MGNREGS' Beneficiaries and Implementing Agencies about the Planning, Implementation and Shortcomings of the Scheme in Telangana State, India. Int.J.Curr.Microbiol.App.Sci. 7(08): 1282-1294. doi: https://doi.org/10.20546/ijcmas.2018.708.145 